\title{
Conformal window in SU(2) with fundamental fermions
}

\author{
Viljami Leino ${ }^{1, \star}$, Jarno Rantaharju², Teemu Rantalaiho, Kari Rummukainen ${ }^{1}$, Joni Suorsa ${ }^{1}$, \\ Kimmo Tuominen ${ }^{1}$, and Sara Tähtinen ${ }^{1}$ \\ ${ }^{1}$ University of Helsinki and Helsinki Institute of Physics \\ ${ }^{2}$ Duke University
}

\begin{abstract}
We present the updated results of the infrared behavior of the SU(2) model with 6 and 8 fundamental representation fermions. We use the gradient flow method with the Schrödinger functional boundary conditions to measure the running of the coupling in these theories and find fixed points on both. We also measure the mass anomalous dimension from these configurations.
\end{abstract}

\section{Introduction}

The search for phenomenologically viable models for beyond the standard model scenarios has led to a studies of a vacuum phase of $\mathrm{SU}\left(N_{c}\right)$ gauge theories as a function of $N_{f}$ massless flavors of Dirac fermions. At a small $N_{f}$ these theories are confining and break the chiral symmetry analogously to the QCD. On the other hand, at large $N_{f}^{\mathrm{AF}}=11 N_{c} / 2$ the theory loses asymptotic freedom and the Gaussian fixed point is no longer attractive in the ultraviolet. Below $N_{f}^{\mathrm{AF}}$ the theory develops a nontrivial infrared fixed point (IRFP) at which only the mass stays a relevant coupling and all the other operators, including gauge coupling, become irrelevant. While the theory has an IRFP, the theory is conformal i.e. scale invariant and no confinement or chiral symmetry breaking can occur. When the $N_{f}$ is lowered, the IRFP moves to the stronger coupling until at $N_{F}^{C}$ the theory becomes chirally broken. The interval $N_{f}^{C}<N_{f}<N_{f}^{\mathrm{AF}}$ is called the conformal window. The lower edge of conformal window, marked by $N_{f}^{C}$, is of a special interest as the IRFP typically occurs at large coupling mandating the use of non-perturbative methods such as lattice simulations. Over recent years, the infrared behaviour of multiple different models with varying $N_{c}, N_{f}$, and different fermion representations have been heavily studied on the lattice. For recent reviews, see [1, 2].

In this paper we focus on the infrared behavior of $\mathrm{SU}(2)$ gauge theories, with varying number of fundamental representation massless Dirac fermions. With $N_{f}=2$ the chiral symmetry is broken and the theory forms a basic template for a dynamical electroweak symmetry breaking [3]. Likewise, the lattice studies on $N_{f}=4$ indicate the theory to be chirally broken [4]. Different approximations estimate the lower boundary of conformal window to be $N_{f}^{C} \sim 6-8[5,6]$. However, both the $N_{f}=6$ and $N_{f}=8$ have been controversial, with previous studies being inconclusive [4,7-10]. The $N_{f}=10$ was confirmed to have an IRFP in [4] and above $N_{f}=11$ the theory loses its asymptotic freedom.

In this paper we review the results obtained in $[11,12]$. In these studies we see a clear indication for the existence of IRFP in the SU(2) gauge theory for both $N_{f}=6$ and $N_{f}=8$. We also report

^ Speaker, e-mail: viljami.leino@helsinki.fi 
measurements for the gauge invariant quantities, the mass anomalous dimension $\gamma_{m}^{*}$ and the leading irrelevant exponent $\gamma_{g}^{*}$ related to the slope of $\beta$-function.

\section{Lattice setup}

We model the SU(2) gauge theory with varying number of massless Dirac fermions in the fundamental representation using a lattice action:

$$
S=\left(1-c_{g}\right) S_{G}(U)+c_{g} S_{G}(V)+S_{F}(V)+c_{S W} \delta S_{S W}(V),
$$

where the smeared gauge link $V$ is defined by the hypercubic truncated stout smearing (HEX) [13], and the smeared, $S_{g}(V)$, and unsmeared, $S_{G}(U)$, Wilson gauge actions are mixed together with mixing coefficient $c_{g}=0.5$. The clover fermion action, $S_{F}(V)$, is improved to order $O(a), a$ being the lattice spacing, by the use of tree-level Sheikholeslami-Wohlert coefficient $c_{S W}=1$. We use the Schrödinger Functional method [14] with Dirichlet boundary conditions at the temporal boundaries $x_{0}=0, L$.

These boundary conditions allow us to run our simulations at zero quark mass. Thus we define $\kappa_{c}\left(\beta_{L}\right)$ as the value at which the PCAC mass [15] vanishes. We tune the value of $\kappa_{c}\left(\beta_{L}\right)$ on lattices of size $L=24$ for each $\beta_{L}$ in use by doing multiple measurements of the PCAC mass with different values of the hopping parameter $\kappa$ and interpolating to zero mass. We attain an accuracy of $10^{-5}$.

To run our simulations we utilize the hybrid Monte Carlo algorithm with 2nd order Omelyan integrator [16]. The step length is tuned to achieve an acceptance ratio above $85 \%$. We follow the evolution of topological charge during the simulations and make sure to only do analysis on trajectories free of topological freezing. In general we generate $(5-100) \cdot 10^{3}$ trajectories for each combination of $\beta_{L}$ and $L$ used. The comprehensive algorithmic details are described in [11, 17].

\section{Theory}

\subsection{Running of the coupling}

We measure the coupling using the Yang-Mills gradient flow [18, 19]:

$$
\partial_{t} B_{\mu}=D_{v} G_{v \mu},
$$

where the initial condition is $B_{\mu}(x ; t=0)=A_{\mu}(x), D_{\mu}=\partial_{\mu}+\left[B_{\mu}, \cdot\right]$ is the covariant derivative of the field strength tensor $G_{\mu \nu}(x ; t)$ and $t$ is the fictitious flow time. This smearing transformation drives the gauge field towards the minima of Yang-Mills action and continuously removes the UV divergences by smoothing the gauge field. The gradient flow automatically renormalizes gauge invariant objects, allowing us to measure the coupling at a scale $\mu=1 / \sqrt{8 t}$ as:

$$
g_{\mathrm{GF}}^{2}(\mu)=\left.\mathcal{N}^{-1} t^{2}\left\langle E\left(t+\tau_{0} a^{2}\right)\right\rangle\right|_{x_{0}=L / 2, t=1 / 8 \mu^{2}},
$$

where the $\tau_{0}$ is a tunable shift parameter introduced in [20] to reduce the $O\left(a^{2}\right)$ discretization effects. We use the normalization factor $\mathcal{N}$ defined in [21] to match the gradient flow coupling $g_{\mathrm{GF}}^{2}$ to the $\overline{\mathrm{MS}}$ coupling in the tree level. As the translation symmetry is broken by the chosen boundary conditions, the coupling is only measured along the central time slice.

The finite size and cutoff effects in gradient flow coupling depend on the discretizations chosen for the action used for the simulations, the action minimized by the flow, definition of the energy density $E(t)$ and the chosen boundary conditions. Therefore, the scale $\mu$ is commonly limited to a regime where both the cutoff effect and statistical variance, that tends to increases with larger flow times, are 
reasonably small. This is achieved by choosing a dimensionless parameter $c_{t}=\sqrt{8 t} / L=L / \mu[21,22]$, which defines the renormalization scheme.

The evolution of the coupling is then quantified by the step scaling function:

$$
\Sigma(u, L / a, s)=\left.g_{\mathrm{GF}}^{2}\left(g_{0}, s L / a\right)\right|_{g_{\mathrm{GF}}^{2}\left(g_{0}, L / a\right)=u},
$$

which describes the change in the measured coupling $g_{\mathrm{GF}}^{2}$ caused by the increase in the lattice size from $L$ to $s L$. As we use $O(a)$ improved actions, we expect the lowest order discretization effects to be of order $O\left(a^{2}\right)$ and extrapolate the continuum step scaling function with a fit:

$$
\Sigma(u, L / a)=\sigma(u)+c(u)(a / L)^{2} .
$$

In the proximity of an IRFP the continuum step scaling function $\sigma$ can be related to the $\beta$-function as:

$$
\beta(g)=-\mu \frac{d g^{2}}{d \mu}=\gamma_{g}^{*}\left(g^{2}-g_{*}^{2}\right) \approx \frac{g}{2 \ln (s)}\left(1-\frac{\sigma\left(g^{2}, s\right)}{g^{2}}\right) .
$$

This allows us to measure the slope of the $\beta$-function depicting the leading irrelevant exponent $\gamma_{g}^{*}$. However, the existence of an IRFP can introduce scaling violations into the typical Symanzik type improvements, bringing into question the validity of the continuum limit (5) at the IRFP. To check the reliability of the $\gamma_{g}^{*}$ measurement from the slope, we compare it to the alternative finite size scaling method devised in [23-26] and fit:

$$
g_{\mathrm{GF}}^{2}(\beta, L)-g_{*}^{2}=\left[g_{\mathrm{GF}}^{2}\left(\beta, L_{\mathrm{ref}}\right)-g_{*}^{2}\right]\left(\frac{L_{\mathrm{ref}}}{L}\right)^{\gamma_{g}^{*}}
$$

Here $L_{\mathrm{ref}}<L$ is in principle arbitrary reference lattice size, and $\gamma_{g}^{*}$ should be independent of it if $g_{\mathrm{GF}}^{2}$ is sufficiently close to the IRFP.

\subsection{Mass anomalous dimension}

Our choice of boundary conditions allows us to also measure the mass anomalous dimension $\gamma_{m}^{*}$ using two different methods, the mass step scaling method and the spectral density method. In the step scaling method we measure the running of the pseudoscalar density renormalization constant [27, 28] as:

$$
Z_{P}\left(g_{0}, L\right)=\frac{\sqrt{N_{c} f_{1}}}{f_{P}(L / 2)}
$$

and define the $\gamma_{m}^{*}$ from the step scaling function:

$$
\gamma_{m}^{*}(u)=-\frac{\log \sigma_{P}(u, s)}{\log s}, \quad \text { where } \quad \sigma_{P}(u, s)=\left.\lim _{a \rightarrow \infty} \frac{Z_{P}\left(g_{0}, s L / a\right)}{Z_{P}\left(g_{0}, L / a\right)}\right|_{g_{\mathrm{GF}}^{2}\left(g_{0}, L / a\right)=u}
$$

The second way to measure $\gamma_{m}^{*}$ is based on the fact that the scaling of the spectral density of massless Dirac operator is also determined by it. Using the recently introduced stochastic methods it is possible to extract the mode number of Dirac operator $[29,30]$ from its eigenvalue density $\rho(\lambda)$. It is known that in the vicinity of an IRFP the mode number follows a scaling behavior:

$$
v(\Lambda) \equiv 2 \int_{0}^{\sqrt{\Lambda^{2}-m^{2}}} \rho(\lambda) \mathrm{d} \lambda \simeq v_{0}(m)+C\left[\Lambda^{2}-m^{2}\right]^{2 /\left(1+\gamma_{*}\right)} \simeq \Lambda^{4 /\left(1+\gamma_{m}^{*}\right)},
$$

where we have assumed $v_{0}(m)$ and $m^{2}$ to be small due to vanishing PCAC quark masses. 


\section{Measurements}

We study the SU(2) gauge theory with $N_{f}=6$ and $N_{f}=8$. For the $N_{f}=6$ we use the lattice sizes of $L=8,10,12,16,18,20,24,30$ and choose the step-size in Eqs (4) and (9) to be $s=3 / 2$. On the other hand, the $N_{f}=8$ analysis is done with $L=6,8,10,12,16,20,24,32$ and $s=2$.

\subsection{Running of the coupling}

We measure the coupling using the gradient flow (2) method, for which we have chosen the LüscherWeisz action for the flow discretization and the clover discretization for the energy $E(t)$. The scale is set by the choice $c_{t}=0.3$ for $N_{f}=6$ and $c_{t}=0.4$ for $N_{f}=8$. We present the lattice step scaling function (4) for both $N_{f}=6$ and $N_{f}=8$ in Figure 1 and observe that at small couplings the results agree with the perturbative curves until they start to curve towards the IRFP at large couplings. On large couplings, the smallest lattice pair on both $N_{f}=6$ and $N_{f}=8$ seems to have slightly different behavior than the rest of the lattice pairs likely caused by finite size effects. Because of this lattices smaller than $L=10$ are excluded from all further analysis.
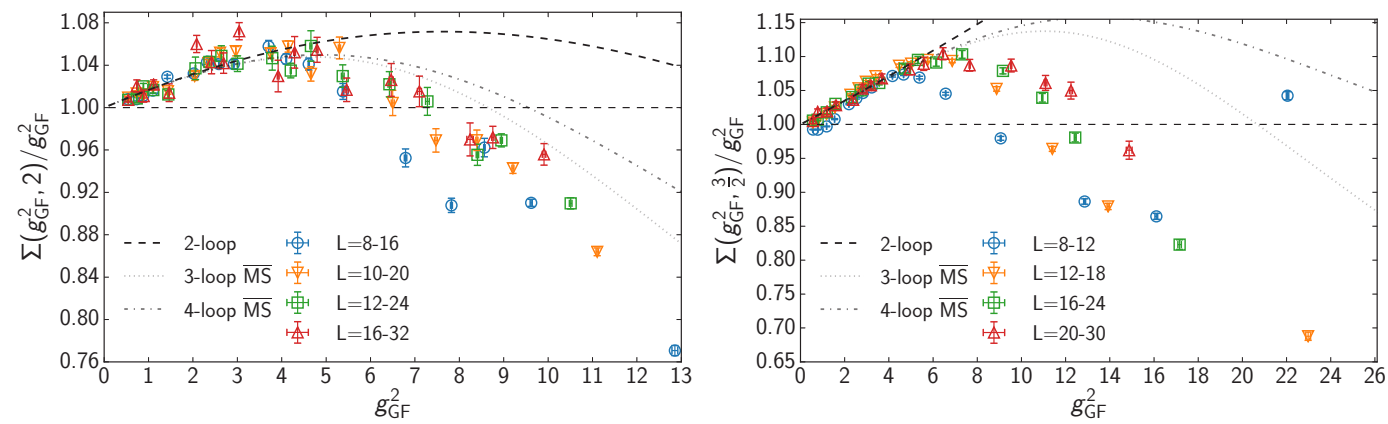

Figure 1. The lattice step scaling function $\Sigma\left(g_{0}^{2}, s\right)$ with $\tau_{0}$-correction for Left: $N_{f}=8$ at $c_{t}=0.4$ and Right: $N_{f}=6$ at $c_{t}=0.3$.
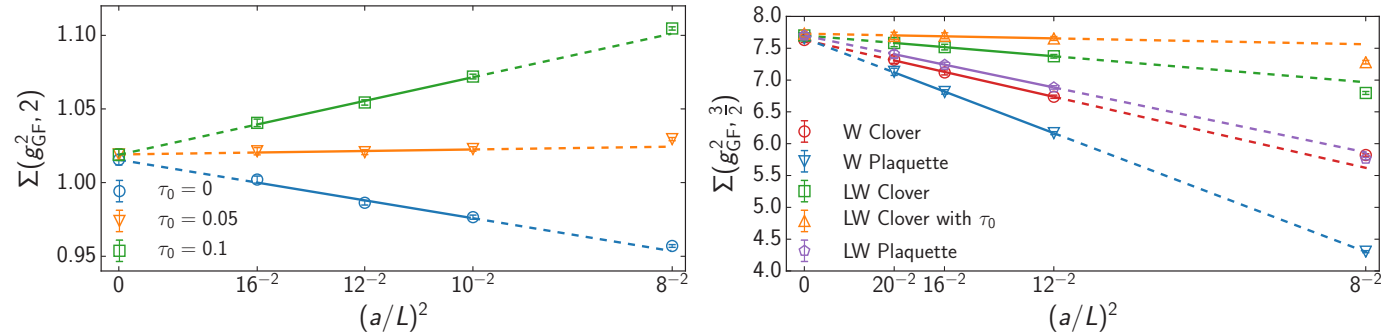

Figure 2. Left: The effect of $\tau_{0}$-correction demonstrated using the continuum limit of the step scaling function in $N_{f}=8$ theory at $g_{\mathrm{GF}}^{2}=1$. Right: The effect of different discretizations to the continuum limit demonstrated using the continuum step scaling function in $N_{f}=6$ theory at $g_{\mathrm{GF}}^{2}=7$. In the legend LW refers to Lüscher-Weisz flow, W refers to Wilson flow and plaquette and clover are the different discretizations for $E(t)$. 

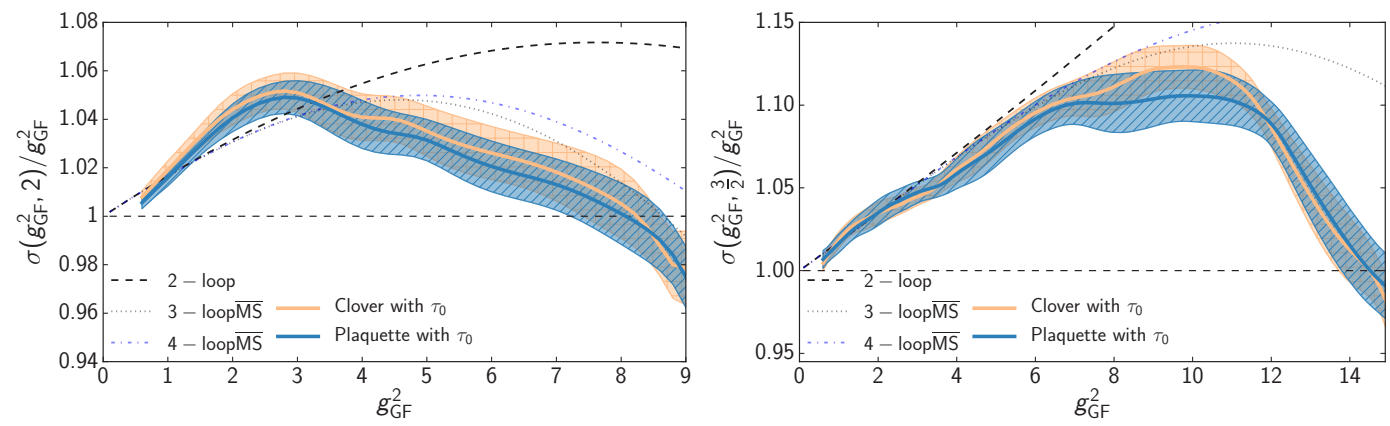

Figure 3. The continuum limit of the step scaling function $\sigma\left(g_{\mathrm{GF}}^{2}, s\right)$ with different discretizations for the $E(t)$ for Left: $N_{f}=8$ at $c_{t}=0.4$ and Right: $N_{f}=6$ at $c_{t}=0.3$. The errors include statistical errors and systematics rising from choice of interpolation function.
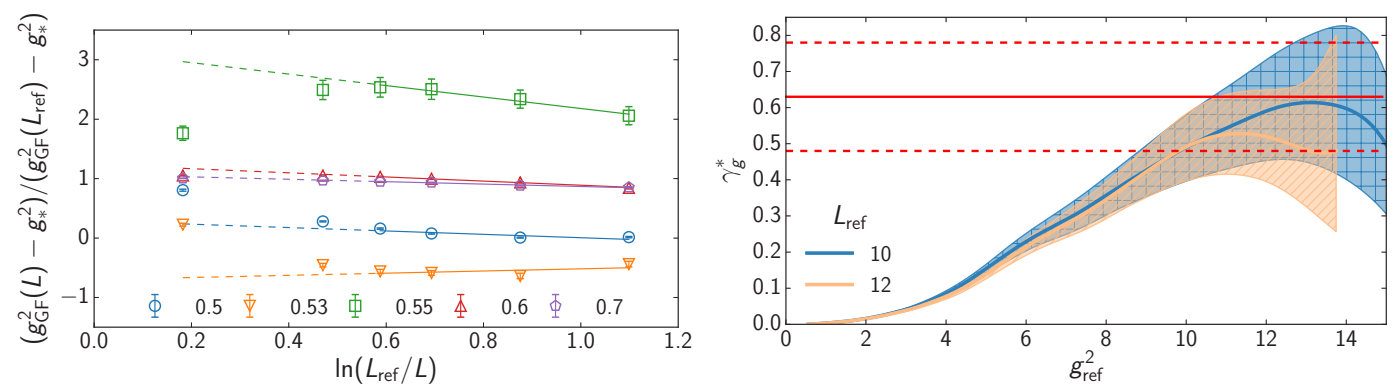

Figure 4. The alternative $\gamma_{g}^{*}$ fit (7), Left: For 5 smallest $\beta$ 's. Right: For all interpolated values of $g_{\text {ref }}^{2}$.

We tune the $\tau_{0}$ correction by minimizing the $O\left(a^{2}\right)$ effects as shown on the left side of Figure 2 . In general, larger couplings $g_{\mathrm{GF}}^{2}$ require larger corrections, but since the $\tau_{0}$ is assumed to be a small correction we have chosen a logarithmic form for the $\tau_{0}$ in order to regulate the strong coupling behavior:

$$
\tau_{0}=0.025 \log \left(1+2 g_{\mathrm{GF}}^{2}\right), \text { for } N_{f}=6 \quad \text { and } \quad \tau_{0}=0.06 \log \left(1+g_{\mathrm{GF}}^{2}\right), \text { for } N_{f}=8
$$

On the right hand side of the Figure 2 we examine the effect of different discretizations to the continuum limit alongside the $\tau_{0}$-correction. Clearly the chosen set of parameters have the smallest discretization effects. Comprehensive analysis for different parameter choices has been done in [11, 12].

We then interpolate the raw couplings, with respect to the bare coupling $g_{0}^{2}=4 / \beta$, using a 9th degree polynomial for the $N_{f}=6$ and using a rational ansatz with 7 th degree polynomial in the numerator and 1 st degree polynomial in the denominator for the $N_{f}=8$. The continuum step scaling function (5) is presented in Figure 3, where we also ensure the consistency of the continuum limit by checking that the two different discretizations of $E(t)$ have the same continuum limit. Both of the theories are found to exhibit an IRFP at: $g_{*}^{2}=14.5(3)_{-1.38}^{+0.41}$ for the $N_{f}=6$ and $g_{*}^{2}=8.24(59)_{-1.64}^{+0.97}$ for the $N_{f}=8$. Here, the first set of errors is statistical and the second set indicates the systematical uncertainty rising from varying different discretizations. 
From the continuum step scaling function we can also measure the slope of beta function (6). In the $N_{f}=8$ no proper measurement can be done due to large errors, but in $N_{f}=6$ we can measure $\gamma_{g}^{*}=0.63(15)_{-0.27}^{+0.28}$ which is consistent with the recent scheme independent estimate $\gamma_{g}^{*}=0.6515$ [31]. For the alternative method (7) we observe high discretization effects, shown on the left of Figure 4, forcing us to only use $L>16$ for the main fit, $L_{\text {ref }}$ varying between 10 and 12 . From the right hand side of the Figure 4, we can see this method gives comparable values at the IRFP, although the signal gets weak closer to the IRFP as indicated by the downwards divergence in the figure.

\subsection{Mass anomalous dimension}

By interpolating pseudoscalar density renormalization constant $Z_{p}(8)$ with a 8th order polynomial for the $N_{f}=6$ and 6th order polynomial for the $N_{f}=8$, we get the continuum limit of the mass step scaling function (9), shown in Figure fig-7. From the figure we observe that the mass step scaling method becomes unstable at a large couplings and no IRFP value for the $\gamma_{m}^{*}$ can be quoted.
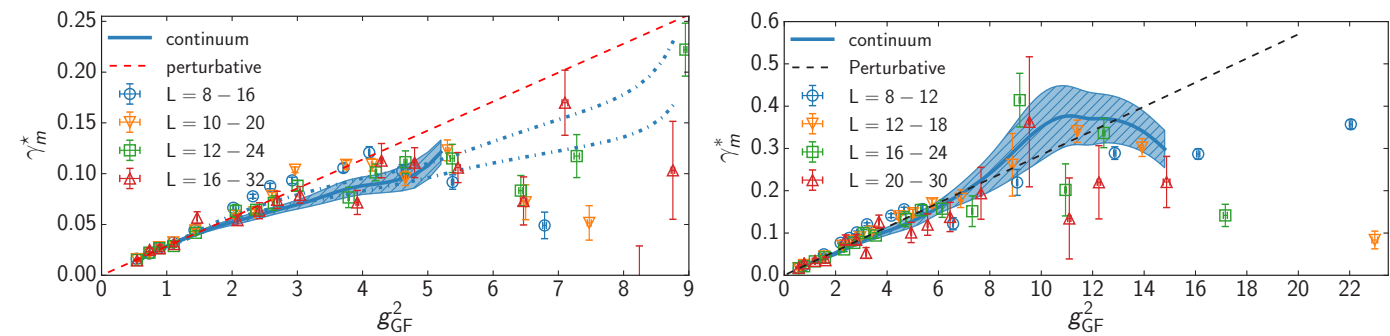

Figure 5. The mass anomalous dimension $\gamma_{m}^{*}$ measurement together with its continuum limit (9) for: Left: $N_{f}=8$ and Right: $N_{f}=6$. In the region with dashed lines, the fit is unacceptably bad.
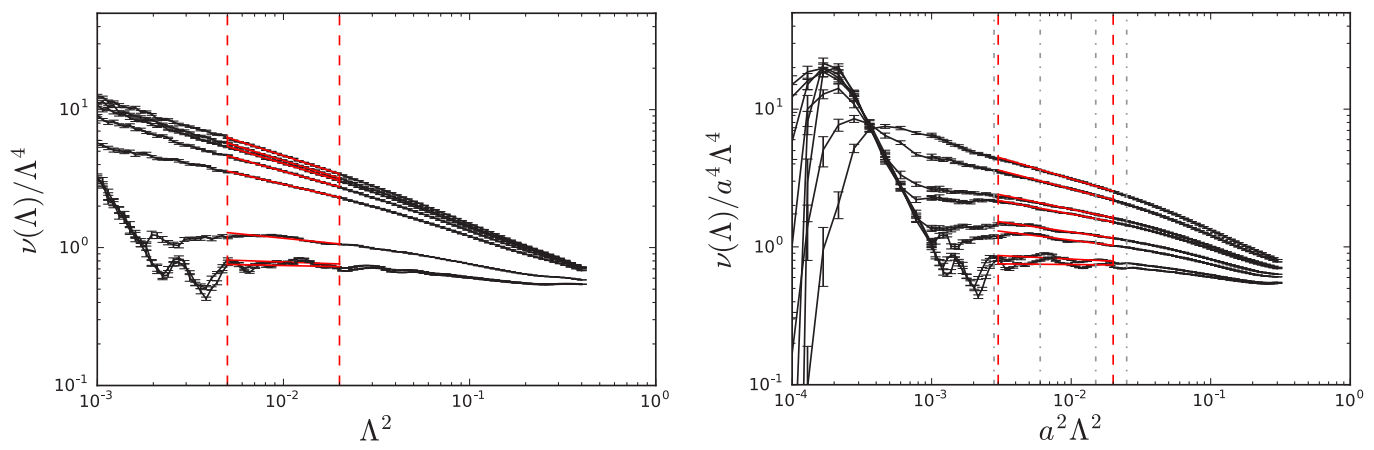

Figure 6. The mode number divided by $a^{4} \Lambda^{4}$ as a function of $a^{2} \Lambda^{2}$ for: Left: $N_{f}=8$ and Right: $N_{f}=6$. The dashed lines indicate the chosen fit range.

To make a more definitive measurement of the $\gamma_{m}^{*}$ we turn our focus to the spectral density method. Taking 10-20 well separated configurations from the step scaling study for each $\beta_{L}$ at the $L=24$ for $N_{f}=6$ and $L=32$ for $N_{f}=8$, we calculate the mode number for 90 values of $\Lambda^{2}$ ranging between 

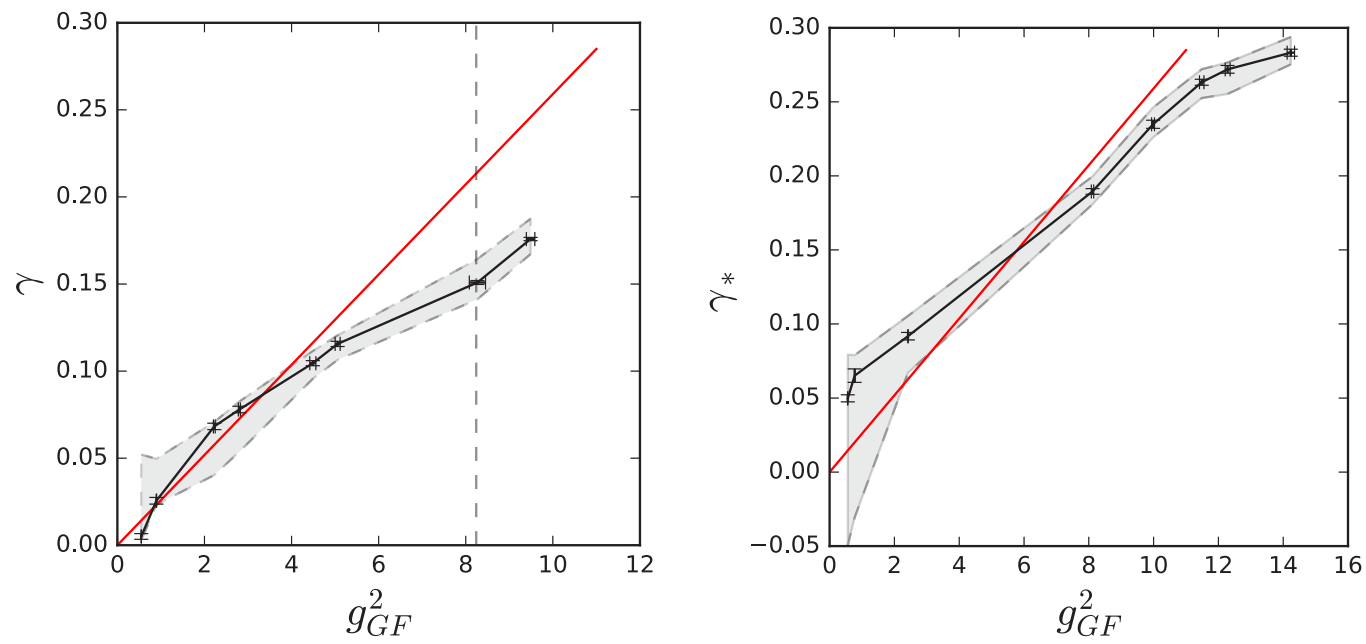

Figure 7. The mass anomalous dimension $\gamma_{m}^{*}$ using the spectral density method (10) for Left: $N_{f}=8$ and Right: $N_{f}=6$. The shaded bands illustrates the uncertainty from varying the fit range by $50 \%$.

$10^{-3}-0.3$. We measure the $\gamma_{m}^{*}$ from the mode number by doing the scaling fit (10), shown in Figure 6 . The right scaling is observed at the strong coupling, however, at small coupling the low eigenvalues appear at discrete energies, which can be seen as bumps in the figure. The right fit range is determined by cross referencing both perturbation theory and step scaling method at low couplings.

In Figure 7 we present the final measurement of $\gamma_{m}^{*}$, where the shaded bands show the errors arising from varying the fit range in Figure 6. As the bumps at the low coupling make the fit harder, the largest uncertainty from this method arises from the low couplings. On the other hand, on large couplings this method turns out to be extremely reliable. Therefore these results complement the results from the step scaling method perfectly and we can measure: $\gamma_{m}^{*}=0.283(2)_{-0.01}^{+0.01}$ for $N_{f}=6$ and $\gamma_{m}^{*}=0.15(2)$ for $N_{f}=8$ at the IRFP.

\section{Conclusions}

We have studied the running coupling in the SU(2) lattice gauge theory with 6 and 8 fermions in the fundamental representation and showed that both theories develop an infrared fixed point at $g_{*}^{2}=$ $14.5(3)_{-1.38}^{+0.41}$ and $g_{*}^{2}=8.24(59)_{-1.64}^{+0.97}$ for for $N_{f}=6$ and $N_{f}=8$ respectively. The existence of an IRFP is compatible with our study of the mass spectrum in SU(2) theories [32]. We have measured the scheme independent quantities $\gamma_{m}^{*}=0.283(2)_{-0.01}^{+0.01}$ for $N_{f}=6$ and $\gamma_{*}=0.15(2)$ for $N_{f}=8$, and $\gamma_{g}^{*}=0.63(15)_{-0.27}^{+0.28}$ for $N_{f}=6$.

\section{Acknowledgments}

This work is supported by the Academy of Finland grants 267286 and 267842. V.L. and J.S. acknowledge the funding by Jenny and Antti Wihuri foundation and T.R. and S.T. are funded by the Magnus Ehrnrooth foundation. The simulations were performed at the Finnish IT Center for Science (CSC) in Espoo, Finland, on the Fermi supercomputer at Cineca in Bologna, Italy, and on the K computer at Riken AICS in Kobe, Japan. 


\section{References}

[1] C. Pica, PoS LATTICE2016, 015 (2016), 1701.07782

[2] B. Svetitsky, Looking behind the Standard Model with lattice gauge theory (2017), in Proceedings, 35th International Symposium on Lattice Field Theory (Lattice2017): Granada, Spain, to appear in EPJ Web Conf., 1708.04840

[3] A. Hietanen, R. Lewis, C. Pica, F. Sannino, JHEP 07, 116 (2014), 1404. 2794

[4] T. Karavirta, J. Rantaharju, K. Rummukainen, K. Tuominen, JHEP 05, 003 (2012), 1111.4104

[5] F. Sannino, K. Tuominen, Phys. Rev. D71, 051901 (2005), hep-ph/0405209

[6] D.D. Dietrich, F. Sannino, Phys. Rev. D75, 085018 (2007), hep-ph/0611341

[7] H. Ohki, T. Aoyama, E. Itou, M. Kurachi, C.J.D. Lin, H. Matsufuru, T. Onogi, E. Shintani, T. Yamazaki, PoS LATTICE2010, 066 (2010), 1011. 0373

[8] F. Bursa, L. Del Debbio, L. Keegan, C. Pica, T. Pickup, Phys. Lett. B696, 374 (2011), 1007.3067

[9] M. Hayakawa, K.I. Ishikawa, S. Takeda, M. Tomii, N. Yamada, Phys. Rev. D88, 094506 (2013), 1307.6696

[10] T. Appelquist, R. Brower, M. Buchoff, M. Cheng, G. Fleming, J. Kiskis, M. Lin, E. Neil, J. Osborn, C. Rebbi et al., Phys. Rev. Lett. 112, 111601 (2014), 1311.4889

[11] V. Leino, J. Rantaharju, T. Rantalaiho, K. Rummukainen, J.M. Suorsa, K. Tuominen, Phys. Rev. D 95, 114516 (2017), 1701.04666

[12] V. Leino, K. Rummukainen, J.M. Suorsa, K. Tuominen, S. Tähtinen (2017), 1707.04722

[13] S. Capitani, S. Durr, C. Hoelbling, JHEP 11, 028 (2006), hep-lat/0607006

[14] M. Luscher, P. Weisz, U. Wolff, Nucl. Phys. B359, 221 (1991)

[15] M. Luscher, P. Weisz, Nucl. Phys. B479, 429 (1996), hep-lat/9606016

[16] I.P. Omelyan, I.M. Mryglod, R. Folk, Computer Physics Communications 151, 272 (2003)

[17] J. Rantaharju, T. Rantalaiho, K. Rummukainen, K. Tuominen, Phys. Rev. D93, 094509 (2016), 1510.03335

[18] M. Luscher, Commun. Math. Phys. 293, 899 (2010), 0907 . 5491

[19] M. Lüscher, JHEP 08, 071 (2010), [Erratum: JHEP03,092(2014)], 1006.4518

[20] A. Cheng, A. Hasenfratz, Y. Liu, G. Petropoulos, D. Schaich, JHEP 05, 137 (2014), 1404.0984

[21] P. Fritzsch, A. Ramos, JHEP 10, 008 (2013), 1301 . 4388

[22] Z. Fodor, K. Holland, J. Kuti, D. Nogradi, C.H. Wong, JHEP 11, 007 (2012), 1208 . 1051

[23] T. Appelquist, G.T. Fleming, E.T. Neil, Phys. Rev. D79, 076010 (2009), 0901 . 3766

[24] T. DeGrand, A. Hasenfratz, Phys. Rev. D80, 034506 (2009), 0906. 1976

[25] C.J.D. Lin, K. Ogawa, A. Ramos, JHEP 12, 103 (2015), 1510. 05755

[26] A. Hasenfratz, D. Schaich (2016), 1610. 10004

[27] S. Capitani, M. Luscher, R. Sommer, H. Wittig (ALPHA), Nucl. Phys. B544, 669 (1999), hep-lat/9810063

[28] M. Della Morte, R. Hoffmann, F. Knechtli, J. Rolf, R. Sommer, I. Wetzorke, U. Wolff (ALPHA), Nucl. Phys. B729, 117 (2005), hep-lat/0507035

[29] L. Giusti, M. Luscher, JHEP 03, 013 (2009), 0812 . 3638

[30] A. Patella, Phys. Rev. D84, 125033 (2011), 1106. 3494

[31] T.A. Ryttov, R. Shrock (2017), 1701.06083

[32] A. Amato, T. Rantalaiho, K. Rummukainen, K. Tuominen, S. Tähtinen, PoS LATTICE2015, 225 (2016), 1511.04947 